\title{
Vom Wissen und Schaffen des Rechts im Alten Orient
}

Die communis opinio in der Rechtsgeschichte negiert die Existenz einer Rechtswissenschaft in den (Rechts-)Kulturen des Alten Orients, deren Schriftzeugnisse sich über den Zeitraum vom ausgehenden dritten Jahrtausend v. Chr. bis zum ersten nachchristlichen Jahrhundert und räumlich vom Persischen Golf über Mesopotamien und Südanatolien bis zur Levante erstrecken. ${ }^{\text {I }}$ Wenn überhaupt Ansätze einer theoretischen Reflexion über das Recht in Betracht gezogen werden, so verortet man sie auf einem (rein) praxisbezogenen, technischen Niveau im Kontext der Schreiberausbildung im »Haus, (in) dem Tafeln zugeteilt werden« (sum. é dub-ba-a). ${ }^{2}$ Die Befähigung, Rechtsurkunden ausfertigen oder auch Funktionen in der Verwaltung oder als Richter übernehmen zu können, setzte die Vermittlung auch inhaltlicher Kenntnisse voraus; Instrumente dieser Wissensvermittlung waren in erster Linie Zusammenstellungen wichtiger Vertragsklauseln und Musterverträge sowie Aufzeichnungen fiktiver Rechtsfälle. ${ }^{3}$

Ihre Wirkungsweise und ihr Einfluss auf die Entwicklung des Rechts, wie sie sich in Veränderungen eben der Rechtspraxis manifestiert, lässt sich in Anlehnung an ähnliche Erscheinungen jüngeren rechtsgeschichtlichen Datums mit dem Begriff »Kautelarjurisprudenz « charakterisieren. ${ }^{4}$ Abgesehen von den genannten Beispielen einer »Formularliteratur « ist uns diese, prima facie eher handwerklich als akademisch anmutende, Beschäftigung mit dem Recht ausschließlich in ihren Ergebnissen in Form der überlieferten Vertrags- und Prozessurkunden erhalten, wenn auch in außerordentlich großer Zahl und von überaus reichhaltigem Inhalt, der alle Aspekte des Rechtslebens umfasst. Hingegen bleiben die vorgelagerten - insbesondere intellektuellen - Stadien der Textproduktion mangels Dokumentation weitgehend im Verborgenen. ${ }^{5}$

Dementsprechend war und ist die Forschung in der verhältnismäßig jungen Disziplin der »Keilschriftrechte «, ${ }^{\mathbf{6}}$ und zwar sowohl auf der juristischen wie auf der philologischen Seite dieses in besonderem Maße durch Interdisziplinarität geprägten Gebiets, im Wesentlichen auf die überlieferten Textkorpora und deren Verständnis als solches fokussiert: Differenzieren lässt sich dabei (freilich allenfalls tendenziell) eine - offenkundig romanistisch

I Siehe R. Westbrook, The Character of Ancient Near Eastern Law, in: A History of Ancient Near Eastern Law, ed. by

R. Westbrook, vol. I ( $\left.\mathrm{HdO}_{7} \mathrm{I} / \mathrm{I}\right)$, Leiden, Boston 2003, I-90, $2 \mathrm{I}-$ 24; M. P. Streck, Artikel »Recht. A. In Mesopotamien «, in: Reallexikon der Assyriologie und Vorderasiatischen Archäologie, Bd. I I, Berlin, New York 20062008, 280-285, 282 f.; siehe jüngst aber auch R. Westbrook $†$, The Early History of Law: A Theoretical Essay, in: SZ 127 (20I0) I-I3, insbes. 9 f.; vgl. aber auch etwa F. WIEACKer, Vulgarismus und Klassizismus im Recht der Spätantike, Heidelberg I95 5, 7, der von "vorwissenschaftlichen Rechtskulturen « spricht.

2 Zu juristischen Aspekten der Schreiberausbildung H. NeUMANN, Prozeßführung im
Edubba'a. Zu einigen Aspekten der Aneignung juristischer Kenntnisse im Rahmen des Curriculums babylonischer Schreiberausbildung, in: Zeitschrift für Altorientalische und Biblische Rechtsgeschichte Io (2004) 7I-92.

3 Dazu H. Neumann, Recht im antiken Mesopotamien, in: Die Rechtskulturen der Antike, hg. von U. Manthe, München 2003, 55-I22, S. 64 f.

4 So bei R. HaAse, Einführung in das Studium keilschriftlicher Rechtsquellen, Wiesbaden I965, 6.

5 Ähnliches gilt auch für die griechischen und hellenistischen Rechtskreise, die - selbst in Ansehung der philosophischen Betrachtung rechtlich relevanter Fragen - keine eigene Rechtswissenschaft hervorgebracht haben; den »scientific approach « gegenüber dem Recht darf nach verbreiteter Ansicht die römische Welt als originäre Kulturleistung für sich in Anspruch nehmen; dazu G. THÜR, Recht im antiken Griechenland, in: Rechtskulturen der Antike (Fn. 3) I9I-238, I96.

6 Der Begriff (im Singular) geht zurück auf P. KoschaKer, Keilschriftrecht, in: Zeitschrift der Deutschen Morgenländischen Gesellschaft 89 (N.F. I4, I935) I39, 26; wegen der Differenziertheit der einzelnen keilschriftlich überlieferten Rechtskulturen erscheint der Plural »Keilschriftrechte « vorzugswürdig, so etwa auch G. CARDASCIA, Les droits cunéiformes, Paris I950, I7. Zu den Anfängen der Forschung zum altorientalischen Recht siehe C. WILCKE, Das Recht: Grundlage des sozialen und politischen Diskurses im Alten Orient, in: Das geistige Erfassen der Welt im Alten Orient. Sprache, Religion Kultur und Gesellschaft, hg. von C. Wilcke, Wiesbaden 2007, 209-244, $209 \mathrm{f}$. 


\section{Rechtsgeschichte}

\section{Guido Pfeifer}

\section{Vom Wissen und Schaffen des Rechts im Alten Orient}


7 Überblick bei V. KorošEc, Keilschriftrecht, in: Handbuch der Orientalistik, hg. von B. SPULER, Erste Abteilung, Der nahe und der mittlere Osten, Ergänzungsband III, Orientalisches Recht, Leiden, Köln I964, 49-2 I9, 5I-54.

8 Einen umfassenden Überblick über die Forschungsarbeiten (auch) der rechtshistorischen Altorientalistik bietet die von H. Neumann besorgte jährliche Keilschriftbibliographie; zuletzt Keilschriftbibliographie.67: 2008 (mit Nachträgen aus früheren Jahren), in: Orientalia NS 78 (2009) I"-I 29*.

9 Beispielsweise G. Pfeifer, Vertragsgestaltung und Formularpraxis in Nuzi um I 500 v. Chr. am Beispiel der so genannten Verkaufsadoption, in: Akten des 36 . Deutschen Rechtshistorikertages Halle an der Saale 2006, hg. von R. LIEBERWIRTH, H. LÜCK, BadenBaden 2008, 30I-323.

Io Etwa bei G. Ries, Prolog und Epilog in Gesetzen des Altertums, München I983, 5-74.

I I Zum Beispiel J. Renger, Noch einmal: Was war der "Kodex" Hammurapi - ein erlassenes Gesetz oder ein Rechtsbuch?, in: Rechtskodifizierung und soziale Normen im interkulturellen Vergleich, hg. von H.-J. GeHrKe, Tübingen I994, 27-59.

I2 Überblick über die Literatur bei S. A. JACKson, A Comparison of Ancient Near Eastern Law Collections Prior to the First Millenium B. C., New Jersey 2008, 69II $3,257-276$.

I3 Siehe Neumann 2003 (Fn. 3) 64, der die Verwendung der Rechtssammlungen in den Schreiberschulen impliziert, umgekehrt die Frage nach ihrem Entstehen aber nicht stellt. Zur möglichen Rolle der Rechtsgewohnheit(en) als Hintergrund der Rechtssammlungen siehe G. Pfeifer, Rechtsgewohnheiten in der Perspektive der altorientalischen Rechtsgeschichte, in: $\operatorname{Rg}$ I 7 (20IO) 8I-83.

I4 H. Petschow, Zur Systematik und Gesetzestechnik im Codex Hammurabi, in: Zeitschrift für Assyriologie und Vorderasiatische Archäologie NF 23 (1965) I46ํํํ I72.

geprägte - dogmatische Orientierung an Rechtsinstituten in der ersten Hälfte des zwanzigsten Jahrhunderts ${ }^{7}$ gegenüber vermehrt (archiv-)kontextbezogenen Untersuchungen, die eher politischen und sozio-ökonomischen Aspekten Rechnung tragen, in der Zeit bis dato. ${ }^{8}$ Untersuchungen, die explizit die Produktion von Rechtstexten und ihren methodischen Hintergrund thematisieren, konzentrieren sich wiederum vornehmlich auf technische Fragen. ${ }^{9}$

Eine gewisse Ausnahme hierzu stellen die zahlreichen Beiträge zu den altorientalischen Rechtssammlungen als besonderer Textgattung dar, über deren Natur und Funktion bis heute gestritten wird: Beispielsweise auf der Basis der Kontextualisierung juristischer und nichtjuristischer Bestandteile der Rechtssammlungen selbst $^{\text {Io }}$ oder infolge der Verortung der Rechtssammlungen in außerrechtlichen literarischen Traditionslinien ${ }^{\text {II }}$ werden hier Fragestellungen und Antworten möglich, die gleichsam über Text und Kontext hinausgreifen, wobei das Spektrum der Antworten von der Qualifikation der Rechtssammlungen als autoritativ gesetztes (positives) Recht bis zur Deutung als (bloßer) politischer Propaganda reicht. ${ }^{\mathbf{I 2}}$

Gewissermaßen die media sententia in dieser Diskussion verweist auch hier auf den Kontext der Schreiberschulen, ohne indes den als solchen schlüssigen Konnex zwischen Rechtspraxis und normativer Textproduktion in erschöpfendem Maß deskriptiv ausfüllen zu können. ${ }^{\mathbf{} 3}$ Selbst dezidiert methodisch orientierte Untersuchungen auf diesem Feld ${ }^{\mathbf{I}}$ attestieren der insoweit repräsentierten keilschriftrechtlichen Überlieferung zwar Indizien für eine »juristisch-theoretische Reflexion «, die aber mangels »zeitgenössischer theoretischer Literatur " nicht greifbar sei. ${ }^{15}$ Diese Forschungsperspektive erscheint insoweit konsequent und schlechterdings nicht kritisierbar, als sie auf die greifbare schriftliche Überlieferung rekurriert und spekulative Annahmen weitgehend vermeidet. Ergo: Keine Rechtswissenschaft im Alten Orient!?

Parallelisieren lassen sich diese Beobachtungen mit einem Blick in die allgemeine Assyriologie als der gleichsam geringfügig älteren Halbschwester der Keilschriftrechtsgeschichte: Hier wurde die Frage nach der Wissenschaft im Alten Orient als solcher schon früh gestellt und wird es bis heute. ${ }^{\text {I6 }}$ Mitunter gewann sie nachgerade neuralgische Bedeutung im Sinne einer Standortbestimmung für die sich etablierende Disziplin, etwa im Fall von Benno Landsbergers Entwicklung des Gedankens von der »Eigenbegrifflichkeit

I 5 Petschow (Fn. I4) I72; etwas optimistischer Neumann 2004 (Fn. 2) 92.

I 6 Überblick bei E. CANCIK-KIRSCHBAUM, Gegenstand und Methode: Sprachliche Erkenntnistechniken in der keilschriftlichen Überlieferung Mesopotamiens, in: Writings of Early Scholars in the Ancient Near East, Egypt Rome and Greece, ed. by A. ImHausen,
T. Pommering, Berlin, New York 2OIO, I3-45, I6-I8.

I7 B. LANDSBERger, Die Eigenbegrifflichkeit der babylonischen Welt, in: Islamica 2 (I926) 355372 (Nachdruck mit Nachwort Darmstadt I965, 2. Aufl. I974). Zur Bedeutung der Schrift siehe W. Sallaberger, Benno Landsbergers "Eigenbegrifflichkeit " in wissenschaftsgeschichtlicher Per- 
der babylonischen Welt « ${ }^{17}$ Sehr verkürzt zusammengefasst besagt Landsbergers Idee, dass aus der Struktur der (sumerischen) Sprache ein »Ordnungsbegriff ${ }^{\mathrm{I} 8}$ abgeleitet werden könne, welcher den altmesopotamischen Kulturen Wissenschaft in Form lexikalischer Listen ${ }^{19}$ ermöglicht habe. Eine solche stellt etwa auch die zweisprachige (sumerisch-akkadische) Serie ana ittišu dar, die benannt nach den Anfangsworten (»auf Abruf zur vereinbarten Zeit«) - eine Zusammenstellung von Vertragsklauseln und rechtlich relevanter Terminologie enthält. ${ }^{20}$

Die wissenschaftstheoretische Perspektive Landsbergers hat sich zwar nicht durchgesetzt; zudem zeigte sich in der Assyriologie in der zweiten Hälfte des zwanzigsten Jahrhunderts - ähnlich wie in anderen Fächern, nicht zuletzt auch der Rechtsgeschichte - eine Abkehr von ausgreifenden Kulturtheorien und eine Tendenz zum genuin philologischen Arbeiten. ${ }^{2 \mathrm{I}}$ Trotz einer daraus resultierenden grundsätzlichen Skepsis bleibt die methodische Herausforderung offenkundig, die keilschriftlich überlieferten Wissenskulturen adäquat zu beschreiben, nicht zuletzt in Abgrenzung zum modernen Wissenschaftsbegriff, der durch die Referenz gegenüber der griechischen Antike geprägt ist. ${ }^{22}$

Im Zusammenhang mit den jüngsten Arbeiten zu diesem Themenkomplex, ${ }^{23}$ zu denen auch zwei aktuelle Frankfurter Forschungsprojekte im Rahmen des Exzellenzclusters 243 "Die Herausbildung normativer Ordnungen « (Forschungsfeld 2: Die Geschichtlichkeit normativer Ordnungen) gehören, ${ }^{24}$ fällt auf, dass dem Recht jedenfalls keine zentrale Rolle beigemessen zu werden scheint. ${ }^{25}$ Dies wiederum ist zumindest insofern bemerkenswert, als die Zahl der Texte, die in einem engeren oder weiteren $\mathrm{Zu}$ sammenhang mit dem Recht gesehen werden kann, etwa drei Viertel der gesamten keilschriftlichen Überlieferung ausmacht, ${ }^{26}$ die Rechtstexte mithin einen Faktor der altorientalischen Wissenskultur darstellen, der schlechterdings nicht zu ignorieren ist. Dass die Berücksichtigung von Rechtstexten für die Beschreibung des Umgangs mit Wissen nachgerade wegweisend sein könnte, zeigt der Versuch, die Verarbeitung von Informationen im Rahmen von Wissenstexten als prozedurale Struktur zu beschreiben. ${ }^{27}$ Zugleich erscheint die Kontextualisierung der Überlieferung unterschiedlicher Disziplinen, wie etwa Astronomie, Divination, Mathematik, Medizin, Recht, etc., als viel versprechende Alternative zur isolierten Betrachtung des jeweiligen Textmaterials. ${ }^{28}$

spektive, in: Das geistige Erfassen (Fn. 6) 63-82, 64-66.

I8 Sein Schüler Wolfram von Soden ersetzt diesen Terminus 1936 durch »Ordnungswillen «, vgl. W. v. SoDEn, Leistung und Grenze sumerischer und babylonischer Wissenschaft, in: Die Welt als Geschichte 2 (I936) 4II-464, 509557, 4I9, vollständiges Zitat bei SALLABERGer (Fn. I7) 75 f. mit Fn. 45 . i9 $\mathrm{Zu}$ diesen A. Cavigneaux, Art. "Lexikalische Listen «, in: Reallexikon der Assyriologie und Vorderasiatischen Archäologie, hg. von D. O. EdZard, Bd. 6, Berlin, New York I980-1983, 609-64I sowie jüngst D. O. EdZard †, Die altmesopotamischen lexikalischen Listen - verkannte Kunstwerke?, in: Das geistige Erfassen (Fn. 6) I7-26.

20 Siehe Neumann 2003 (Fn. 3) 64.
2I Siehe den Rückblick bei SALlaBERGER (Fn. I7) 78 .

22 Dazu Cancik-Kirschbaum (Fn. I6) I3-2I.

23 Siehe die (teilweise bereits zitierten) Beiträge in den Sammelbänden Das geistige Erfassen (Fn. 6) sowie Writings of Early Scholars (Fn. I6).

24 A. Warner (Imhausen), Mathematik und die Etablierung normativer Ordnungen in antiken Kulturen: Ägypten und Mesopotamien im Vergleich, siehe http:// www.normativeorders.net/de/ forschung/forschungsprojekte/ 300-mathematik-und-die-etablierung-normativer-ordnungen-in-antiken-kulturen-aegypten-und-mesopotamien-im-vergleich [I 2.05.2OII] und A. WARNER (IMHAUSEN), D. BAWANYPECK, Zur Funktion der Kanonisierung bei der Wissensüberlieferung in Mesopotamien, siehe http:// www.normativeorders.net/de/ forschung/forschungsprojekte/ 30I-zur-funktion-der-kanonisierung-bei-der-wissensueberlieferung-in-mesopotamien [I2.05.20II].

25 Ausnahmen stellen dar Wilcke (Fn. 6) 209-244 sowie E. DoMBRADI, Das altbabylonische Urteil. Mediation oder res iudicata? Zur Stellung des Keilschriftrechts zwischen Rechtsanthropologie und Rechtsgeschichte, in: Das geistige Erfassen (Fn. 6) 245-279; beide Beiträge betreffen aber die soziale Funktion des Rechts bzw. dogmatische Fragen, nicht die Wissensvermittlung auf dem Gebiet des Rechts.

26 Überblick bei Westbrook (Fn. I) 5-I 2.

27 So J. Ritter, Reading Strasbourg 368: A Thrice-Told Tale, in: History of Science, History of Text, ed. by K. Chemla, Dordrecht 2004, I77-200. Zur prozeduralen Technik der Wissensverarbeitung siehe auch Cancik-Kirschbaum (Fn. I6) I9.

28 Diesem Aspekt hat sich der vom 2. bis 4. Juli 2004 von Annette Warner (Imhausen) am Forschungskolleg Humanwissenschaften in Bad Homburg v. d. H. veranstaltete Workshop »Prozedurentexte in Ägypten und Mesopotamien " gewidmet. 
Für den Rechtshistoriker im Bereich der Keilschriftrechte ergeben sich daraus gleich eine ganze Reihe von Fragestellungen, die zum einen erhebliche Schwierigkeiten aufwerfen, zum anderen aber durchaus Chancen für einen Zuwachs an Erkenntnis bieten können: Die größten Probleme bereitet das bereits erwähnte Fehlen rechtlicher Reflexion auf einer Meta-Ebene; zusätzlicher Aufschluss über die Behandlung rechtlichen Wissens kann daher allein auf der Grundlage der (Neu-)Auswertung des überlieferten Textmaterials gewonnen werden. Anknüpfend hieran sind jedoch zumindest Präzisierungen hinsichtlich der Beantwortung alter und neuer Fragen möglich, etwa bei der funktionalen Deutung konkreter Texte, ${ }^{29}$ bezüglich der inneren Struktur einzelner Textgattungen ${ }^{30}$ oder im Hinblick auf die Ausbildung einer eigenen Rechtssprache. ${ }^{3 \text { I }}$ Im Hinblick auf die Rechtssammlungen wäre gar eine Art Textstufenforschung ${ }^{32}$ zu erwägen, die eine Annäherung an die Entstehungsumstände der altorientalischen »Codices « ermöglichen könnte. ${ }^{33}$ Angesichts dieses bei weitem noch nicht ausgeschöpften Potentials keilschriftrechtsgeschichtlicher Forschung wird die eingangs zitierte Negation einer altorientalischen Rechtswissenschaft zusehends relativiert: Je deutlicher sich die Konturen des weiten Felds zwischen theoretischem Wissen und handwerklicher Praxis des Rechts zeichnen lassen, desto klarer wird das Bild von den Anfängen unserer Profession und damit zugleich die Semantik ihrer Bezeichnung.

Guido Pfeifer

29 So für die Interpretation (fiktiver) Prozessurkunden und ihrer Rolle bei der inhaltlichen Wissensvermittlung im Hinblick auf die Prozessführung NeUMANN 2004 (Fn. 2) 78-9I.

30 Gegenstand etwa des (unveröffentlichten) Abendvortrags d. Verf. im Rahmen des Sommerkurses des MPIeR am I4. Juli 20Io, Von Prozeduren und Prozessen in altbabylonischer Zeit (leicht modifiziert in englischer Sprache: Procedure and proceeding in the Old Babylonian period, Gainesville, FL (USA), 20. Oktober 20ıо, Levin College of Law, University of Florida).

3 I Dazu Streck (Fn. I) $282 \mathrm{f}$.

32 In (begrifflicher) Anlehnung an

F. WIEACKer, Textstufen klassischer Juristen, Göttingen I960 (Nachdruck I975).

33 Exemplarisch bereits P. KoscHAKER, Rechtsvergleichende Studien zur Gesetzgebung Hammurapis, Königs von Babylon, Leipzig I9I7, 26-33 zur Abfassung des
\I25 CH. Koschakers "Interpolationsverdacht « (ebd., 33) wurde drei Jahrzehnte später durch die Entdeckung der Gesetze von Ešnunna bestätigt, deren $\mathbb{S} \mathbb{S} 36$ und 37 die von Koschaker rechtsvergleichend rekonstruierte ältere Textstufe beinhalten. Während das Depositenrecht des $\mathrm{CH}$ seitdem immer wieder Gegenstand der Betrachtung war - siehe etwa E. Отто, Die rechtshistorische
Entwicklung des Depositenrechts in altorientalischen und altisraelitischen Rechtskorpora, in: SZ RA I05 (I988) I-3 I (Io-I6 zu Koschaker); jüngst auch G. PfeIfer, Antwort auf Philipp Scheibelreiter, in: Symposion 2009, hg. von G. Thür, Wien 2010, 377-382-, ist die Frage nach der Verwandtschaft der beiden Rechtssammlungen jedenfalls nicht grundsätzlich geklärt worden. 